\title{
Distributed Protocols for Ensuring Both Coverage and Connectivity of a Wireless Sensor Network
}

\author{
Chi-Fu Huang, Yu-Chee Tseng, and Hsiao-Lu Wu \\ National Chiao-Tung University
}

\begin{abstract}
Wireless sensor networks have attracted a lot of attention recently. Such environments may consist of many inexpensive nodes, each capable of collecting, storing, and processing environmental information, and communicating with neighboring nodes through wireless links. For a sensor network to operate successfully, sensors must maintain both sensing coverage and network connectivity. This issue has been studied in [Wang et al. 2003; Zhang and Hou 2004a], both of which reach a similar conclusion that coverage can imply connectivity as long as sensors' communication ranges are no less than twice their sensing ranges. In this paper, without relying on this strong assumption, we investigate the issue from a different angle and develop several necessary and sufficient conditions for ensuring coverage and connectivity of a sensor network. Hence, the results significantly generalize the results in [Wang et al. 2003; Zhang and Hou 2004a]. This work is also a significant extension of our earlier work [Huang and Tseng 2003; Huang et al. 2004], which addresses how to determine the level of coverage of a given sensor network, but does not consider the network connectivity issue. Our work is the first work allowing an arbitrary relationship between sensing ranges and communication distances of sensor nodes. We develop decentralized solutions for determining, or even adjusting, the levels of coverage and connectivity of a given network. Adjusting levels of coverage and connectivity is necessary when sensors are overly deployed, and we approach this problem by putting sensors to sleep mode and tuning their transmission powers. This results in prolonged network lifetime.
\end{abstract}

Categories and Subject Descriptors: C.2.2 [Computer-Communication Networks]: Network Protocols-Applications; C.3 [Special-Purpose and Application-Based Systems]: —Real-time and embedded systems

General Terms: Algorithms, Design, Performance

Additional Key Words and Phrases: ad hoc network, coverage, connectivity, energy conservation, power control, sensor network, wireless network

\section{INTRODUCTION}

The rapid progress of wireless communication and embedded micro-sensing MEMS technologies has made wireless sensor networks possible. Such environments may have many inexpensive wireless nodes, each capable of collecting, storing, and processing environmental information, and communicating with neighboring nodes. In the past, sensors are connected by wire lines. Today, this environment is combined with the novel ad hoc networking technology to facilitate inter-sensor communication [Pottie and Kaiser 2000;

Author's address: Department of Computer Science, National Chiao-Tung University, 1001 Ta-Hsueh Road, Hsin-Chu, Taiwan 30050, R.O.C.; E-mail: \{cfhuang, yctseng, hlwu\} @ csie.nctu.edu.tw.

Y.-C. Tsengs research is co-sponsored by Taiwans MoE ATU Program, by NSC under grant numbers 93-2752E-007-001-PAE, 95-2623-7-009-010-ET, 95- 2218-E-009-020, 95-2219-E-009-007, 94-2213-E-009- 004, and 94-2219-E-007-009, by MOEA under grant number 94-EC-17-A-04-S1-044, by ITRI, Taiwan, and by Intel Inc. Permission to make digital/hard copy of all or part of this material without fee for personal or classroom use provided that the copies are not made or distributed for profit or commercial advantage, the ACM copyright/server notice, the title of the publication, and its date appear, and notice is given that copying is by permission of the ACM, Inc. To copy otherwise, to republish, to post on servers, or to redistribute to lists requires prior specific permission and/or a fee.

(c) 20YY ACM 0000-0000/20YY/0000-0001 $\$ 5.00$ 
Sohrabi et al. 2000]. The flexibility of installing and configuring a sensor network is thus greatly improved. Recently, a lot of research activities have been dedicated to sensor networks, including design of physical and medium access layers [Shih et al. 2001; Woo and Culler 2001; Ye et al. 2002] and routing and transport protocols [Braginsky and Estrin 2002; Ganesan et al. 2001; Heinzelman et al. 2000]. Localization and positioning applications of wireless sensor networks are discussed in [Bahl and Padmanabhan 2000; Savvides et al. 2001; Tseng et al. 2003].

Since sensors may be spread in an arbitrary manner, a fundamental issue in a wireless sensor network is to ensure coverage and connectivity. Given a sensor network, the coverage issue is concerned with how well the sensing field is monitored by sensors. In the literature, this problem has been formulated in various ways. Coverage can be regarded as a metric to evaluate the quality of service (surveillance) provided by the network. Between a given pair of points in the sensing field, some works focus on finding a path connecting these two points which is best or worst monitored by sensors when an object traverses along the path [Li et al. 2003; Meguerdichian et al. 2001; Meguerdichian et al. 2001; Veltri et al. 2003]. In [Huang and Tseng 2003; Huang et al. 2004], the coverage problem is formulated as one of determining if a 2D/3D sensing field area is sufficiently $k$-covered, i.e., each point in the field is within the sensing ranges of at least $k$ sensors. The proposed approach looks at how the perimeter of each sensor's sensing range is covered, thus leading to efficient polynomial-time algorithms. On the other hand, some works are targeted at particular applications (such as energy conservation [Abrams et al. 2004; Tian and Georganas 2003; Yan et al. 2003]), but the central idea is still related to the coverage issue.

The connectivity issue is concerned with the diversity of communication paths between sensors. This would affect network robustness and communication performance. The GAF protocol [Xu et al. 2001] aims to extend the network lifetime by turning off redundant nodes while keeping the same level of routing fidelity, which is defined as uninterrupted connectivity between communicating nodes. GAF imposes a virtual grid on the network and nodes in the same grid coordinate with each other to determine who can sleep and how long. Reference [Chen et al. 2002] presents a connectivity-maintaining protocol, SPAN, which can turn off unnecessary nodes such that all active nodes are connected through a communication backbone and all inactive nodes are directly connected to at least one active node. Maintaining a network connected is also a basic requirement of works targeted at topology control, which is to adjust sensors' transmission power for energy efficiency and collision avoidance [Burkhart et al. 2004; Li and Hou 2004; Wattenhofer et al. 2001].

In this work, we study the relationship between sensing coverage and communication connectivity of a sensor network. Reference [Wang et al. 2003] proposes a coverage determination algorithm by looking at how intersection points between sensors' sensing ranges are covered by their neighbors, and claims that coverage can imply connectivity as long as sensors' communication ranges are no less than twice their sensing ranges. A Coverage Configuration Protocol (CCP) that can provide different degrees of coverage and meanwhile maintain communication connectivity is presented. If the communication ranges are less than twice the sensing ranges, [Wang et al. 2003] proposes to integrate CCP with SPAN [Chen et al. 2002] to provide both sensing coverage and communication connectivity. A similar result is also drawn in [Zhang and Hou 2004a], and thus only the coverage problem is addressed. A decentralized density control algorithm called Optimal Geographical Density Control $(O G D C)$ is then proposed to reduce the number of working nodes to cover 
the network.

It is clear that the results in [Wang et al. 2003; Zhang and Hou 2004a] are not applicable when some sensors' communication ranges are less than twice their sensing ranges even though others are not. Also, both [Wang et al. 2003; Zhang and Hou 2004a] assume that all sensors have the same sensing ranges. In this paper, we relax these constrains and show necessary and/or sufficient conditions for a sensor network to be $k$-covered and $k$ connected, and to be $k$-covered and 1-connected. Hence, the results in [Wang et al. 2003; Zhang and Hou 2004a] can be regarded as special cases of what proposed in this paper. Based on these conditions, we then develop decentralized solutions for determining, or even adjusting, the levels of coverage and connectivity of a given network. This results in prolonged network lifetime. As far as we know, no result has addressed the combined issues of coverage, connectivity, power management, and power control under a single framework as is done in this work. The ability of adjusting the levels of coverage and connectivity makes management of the network more flexible. In emergency applications, keeping the network 1-covered and 1-connected may be sufficient. However, when emergency occurs, higher coverage and connectivity may be needed in an on-the-fly manner. For auto-configuration purpose, given an arbitrarily deployed sensor network, we can first calculate the coverage and connectivity levels of the network. If the coverage or connectivity level exceeds our expectation, we can make adjustment using the proposed coverage and connectivity selection protocols to prolong the network lifetime without reducing the sensing and communicating capabilities of the network. This work is a significant extension of our earlier work [Huang and Tseng 2003; Huang et al. 2004], which addresses how to determine the level of coverage of a given sensor network, but does not consider the network connectivity issue. Our work is the first work allowing an arbitrary relationship between sensing ranges and communication distances of sensor nodes. Information about the difference of sensor' sensing ranges is discussed in [Zhang and Hou 2004b].

Some works also consider the coverage and connectivity issue, but have different assumptions or applications. Reference [Shakkottai et al. 2003] considers a grid-based network consisting of sensors which may fail probabilistically and investigates the coverage, connectivity, and diameter of the network. Reference [Inanc et al. 2003] studies the problem of minimizing energy consumption by suspending sensors' sensing and communication activities according to a Markovian stochastic process and meanwhile ensuring communication connectivity and sensing coverage. However, the definitions of "event coverage" and "path connectivity" distinguish from our goals. Given a spatial query requesting for data of interest in a geographical region, the goal of [Gupta et al. 2003] is to select the smallest subset of sensors which are connected and are sufficient to cover the region. The proposed solution is a greedy algorithm which recurrently selects a path of sensors that is connected to an already selected sensor until the given query region is completely covered.

This paper is organized as follows. Section 2 gives some preliminaries. Several conditions for coverage and connectivity are presented in Section 3. Decentralized coverageand-connectivity determination and adjustment protocols are developed in Section 4. Section 5 presents our simulation results. Section 6 draws our conclusions and future work.

\section{PRELIMINARIES AND PROBLEM STATEMENT}

We are given a set of sensors, $S=\left\{s_{1}, s_{2}, \ldots, s_{n}\right\}$, in a two-dimensional area $A$. Each sensor $s_{i}, i=1 \ldots n$, is located at a known coordinate $\left(x_{i}, y_{i}\right)$ inside $A$ and has a sensing 


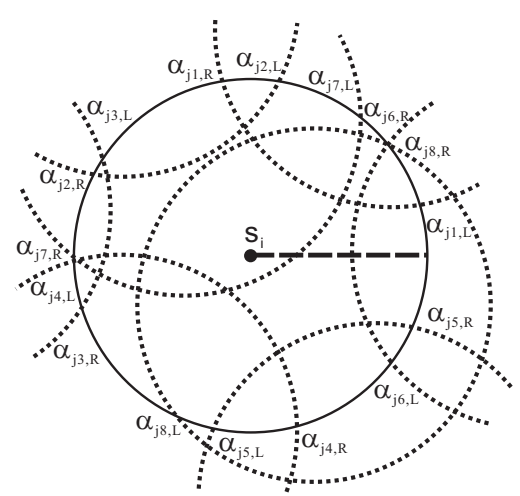

(a)

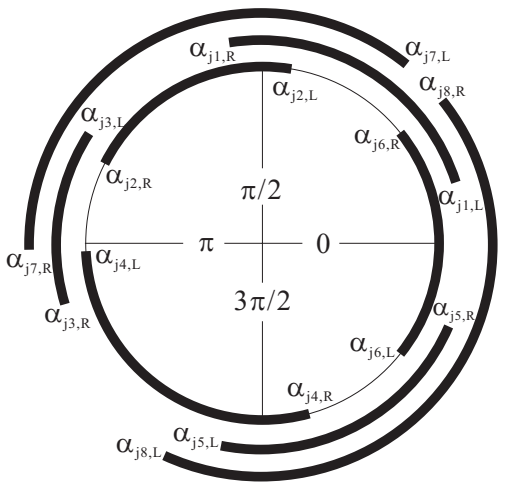

(b)

Fig. 1. Determining the perimeter coverage of a sensor $s_{i}$.

distance of $r_{i}$ and a communication distance of $c_{i}$. So, $s_{i}$ can detect an object/event located within a distance of $r_{i}$ from itself and talk to another sensor within a distance of $c_{i}$. Note that we make no assumption about the relationship of $r_{i}$ and $c_{i}$. Only bidirectional links are considered. So when two sensors can hear each other, we say that there is a communication link, or simply a link, between them.

Definition 1. A point in $A$ is said to be covered by $s_{i}$ if it is within $s_{i}$ 's sensing range. Given an integer $k$, a point in $A$ is said to be $k$-covered if it is covered by at least $k$ distinct sensors. The sensor network is said to be $k$-covered if every point in $A$ is $k$-covered.

Definition 2. The sensor network is said to be 1-connected if there is at least one path between any two sensors. The sensor network is said to be $k$-connected if there are at least $k$ disjointed paths between any two sensors.

The deployment of sensors is not concerned in our work and we assume the network is 1 -covered at least. We formulate the general form of coverage and connectivity problem as follows.

Definition 3. Given any two integers $k_{s}$ and $k_{c}$, the $k_{s}$-Covered and $k_{c}$-Connected Problem, or the $\left(k_{s}, k_{c}\right)$-CC problem, is a decision problem whose goal is to determine whether the sensor network is $k_{s}$-covered and $k_{c}$-connected.

As far as we know, the general $\left(k_{s}, k_{c}\right)$-CC problem has not been well addressed yet. In [Huang and Tseng 2003], the coverage problem has been solved in an efficient way. Below, we briefly review the result, which will be used as a basis in our derivation. Given any sensor, reference [Huang and Tseng 2003] tries to look at the perimeter that bounds the sensor's sensing range (for convenience, this may be simply referred to as "the perimeter of the sensor"). The algorithm essentially determines the coverage level of the sensing field $A$ by determining the perimeter of each sensor.

Definition 4. Consider any two sensors $s_{i}$ and $s_{j}$. A point $p$ on the perimeter of $s_{i}$ is perimeter-covered by $s_{j}$ if this point is within the sensing range of $s_{j}$, i.e., the distance between $p$ and $s_{j}$ is less than $r_{j}$. A point $p$ on the perimeter of $s_{i}$ is $k$-perimeter-covered if 
it is perimeter-covered by at least $k$ sensors other than $s_{i}$ itself. Sensor $s_{i}$ is $k$-perimetercovered if all points on the perimeter of $s_{i}$ are perimeter-covered by at least $k$ sensors other than $s_{i}$ itself.

THEOREM 1. [Huang and Tseng 2003] The whole network area A is $k$-covered iffeach sensor in the network is $k$-perimeter-covered.

The approach in Theorem 1 looks at how the perimeter of each sensor's sensing range is covered by its neighbors. For each sensor $s_{i}$, it tries to identify all neighboring sensors which can contribute some coverage to $s_{i}$ 's perimeter. Specifically, for each neighboring sensor $s_{j}$, we can determine the angle of $s_{i}$ 's arch, denoted by $\left[\alpha_{j, L}, \alpha_{j, R}\right]$, which is perimeter-covered by $s_{j}$. Placing all angles $\left[\alpha_{j, L}, \alpha_{j, R}\right]$ on $[0,2 \pi]$ for all $\mathrm{j}$ 's, it is easy to determine the level of perimeter coverage of $s_{i}$. For example, Fig. 1(a) shows how $s_{i}$ is covered by its neighbors (shown in dashed circles). Mapping these covered angles in Fig. 1(b), it is easy to decide that $s_{i}$ is 1-perimeter-covered. It is shown in [Huang and Tseng 2003] that Theorem 1 can be converted into an efficient coverage determination algorithm. It is to be noted that its makes no sense to consider the perimeter of a sensor exceeding the sensing field $A$. So we only consider the perimeters of sensors inside $A$. In the extreme case that a sensor's sensing range contains $A$, we simply ignore it and consider that it contributes a coverage of 1 to $A$.

\section{CONDITIONS FOR NETWORK COVERAGE AND CONNECTIVITY}

In this section, we propose theoretical foundations and necessary and/or sufficient conditions to solve the $\left(k_{s}, k_{c}\right)$-CC problem. We make no assumption on the relationship between $r_{i}$ and $c_{i}$ of sensor $s_{i}$. We show conditions for a sensor network to be $k$-covered and $k$-connected, and to be $k$-covered and 1 -connected. We also show under what conditions a sensor network may provide sufficient coverage by multiple connected components.

\subsection{Theoretical Fundamentals}

The definition of perimeter coverage has been proved useful to determine the coverage level of a sensor network in [Huang and Tseng 2003]. However, the network connectivity issue has not been studied. For a sensor network to operate successfully, sensors must maintain both sensing coverage and network connectivity. Below we develop some fundamentals to achieve this goal

Definition 5. Consider any sensor $s_{i}$. The neighboring set of $s_{i}$, denoted as $N(i)$, is the set of sensors each of whose sensing region intersects with $s_{i}$ 's sensing region.

Note that neighbors are concerned with how sensors' coverage areas overlap, and should not be confused with communication links, which are concerned with sensors' transmission distances.

Definition 6. Consider any sensor $s_{i}$. We say that $s_{i}$ is $k$-direct-neighbor-perimetercovered, or $k-D P C$, if $s_{i}$ is $k$-perimeter-covered and $s_{i}$ has a link to each node in $N(i)$. Similarly, we say that $s_{i}$ is $k$-multihop-neighbor-perimeter-covered, or $k$-MPC, if $s_{i}$ is $k$-perimeter-covered and $s_{i}$ has a (single- or multi-hop) path to each node in $N(i)$.

These definitions allow us to derive some coverage and connectivity properties of a network. 


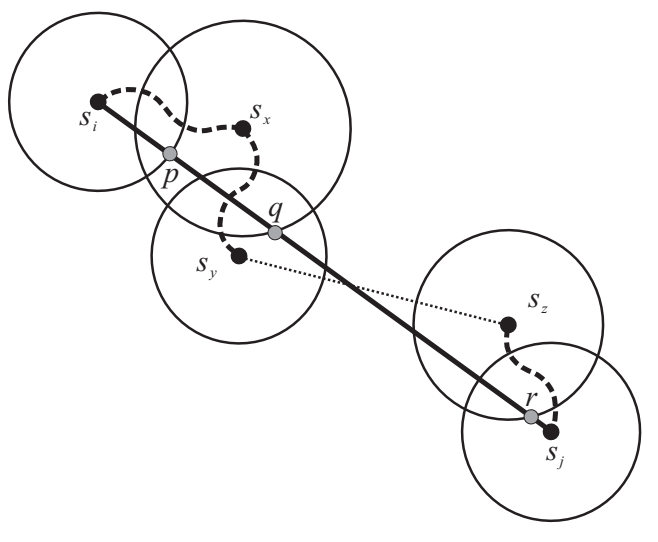

(a)

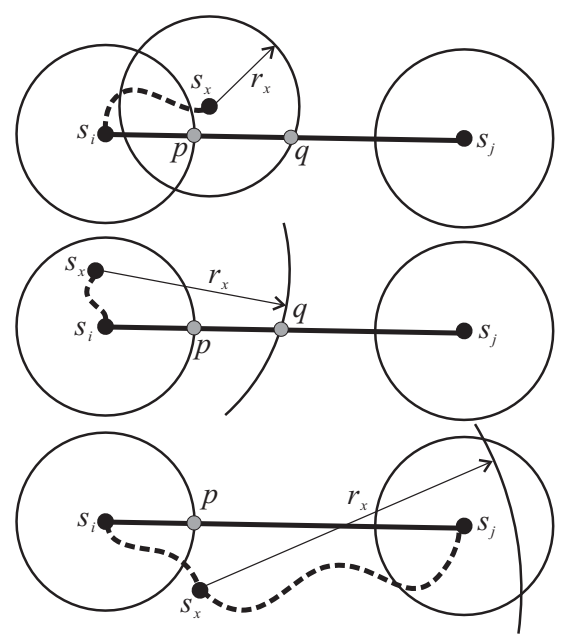

(b)

Fig. 2. Proof of Lemma 1: (a) the path construction, and (b) possible cases of $s_{x}$.

LEMMA 1. Consider any two sensors $s_{i}$ and $s_{j}$. If each sensor in $S$ is 1-MPC, there must exist a communication path between $s_{i}$ and $s_{j}$.

PROOF. This proof is by construction. If $s_{i}$ 's sensing region intersects with $s_{j}$, by Definition 6 , there must exist a path between $s_{i}$ and $s_{j}$, which proves this lemma. Otherwise, draw a line segment $L$ connecting $s_{i}$ and $s_{j}$, as illustrated in Fig. 2(a). Let $L$ intersect $s_{i}$ 's perimeter at point $p$. Since $s_{i}$ is 1-MPC, by Definition 6, there must exist a sensor $s_{x}$ in $N(i)$ which covers $p$ and has a path to $s_{i}$. In addition, either $s_{x}$ must cover $s_{j}$, or $s_{x}$ 's perimeter must intersect $L$ at a point, namely $q$, which is closer to $s_{j}$ than $p$ is. Fig. 2(b) shows several possible combinations of $s_{x}$ and $r_{x}$. In the former case, by Definition 6 , there must exist a path between $s_{x}$ and $s_{j}$, and thus $s_{i}$ and $s_{j}$, which proves this lemma. In the latter case, there must exist another sensor $s_{y}$ in $N(x)$ which covers $q$. We can repeat the above argument until a sensor $s_{z}$ is found which either covers $s_{j}$ or intersects $L$ at a point, say $r$, inside $s_{j}$ 's sensing range. Note that since the number of sensors is finite, the construction must eventually terminate and the path from $s_{i}$ must reach $s_{j}$. Otherwise, the intersection point of $s_{j}$ 's perimeter and $L$ is not covered by any sensor. As a result, there must exist a path between $s_{z}$ and $s_{j}$, which proves this lemma.

THEOREM 2. A sensor network is $k$-covered and 1 -connected iff each sensor is $k$-MPC.

PROOF. For the "if" part, we have to guarantee both the coverage and connectivity. The fact that the network is $k$-covered has been proved by Theorem 1 because each sensor which is $k$-MPC is also $k$-perimeter-covered. In addition, Lemma 1 can guarantee that the network is 1-connected, hence proving the "if" part.

For the "only if" part, we have to show that each sensor is $k$-perimeter-covered and has a path to each sensor whose sensing region intersects with its region. The first concern can be ensured by Theorem 1, while the second concern can be ensured by the fact that the network is 1-connected.

ACM Journal Name, Vol. V, No. N, M 20YY. 


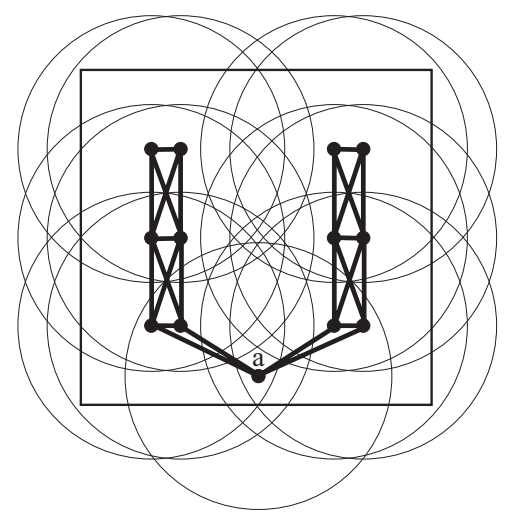

(a)

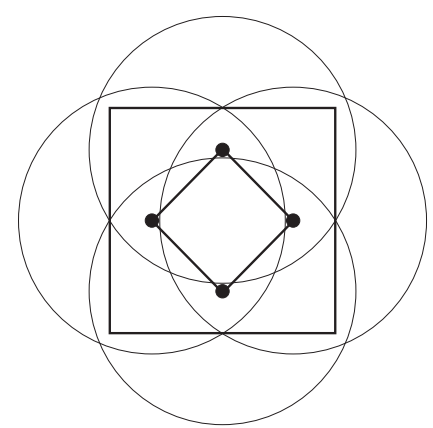

(b)

Fig. 3. Observations of Theorem 2 and Theorem 3: (a) The network is 2-covered and 1connected. The removal of sensor $a$ will disconnect the network, and (b) The network is 2covered and 2-connected but no sensor is 2-DPC. Note that the sensing and communication ranges of each sensor are the same and are represented by circles.

THEOREM 3. A sensor network is $k$-covered and $k$-connected if each sensor is $k$-DPC.

PROOF. Coverage has been guaranteed by Theorem 1 since a sensor which is $k$-DPC is $k$-perimeter-covered by definition. For the connectivity part, if we remove any $k-1$ nodes from the network, it is not hard to see that each of the rest of sensors must remain 1-DPC. This implies that these sensors are also 1-MPC, and by Lemma 1 there must exist a path between any pair of these sensors. As a result, the network is still connected after the removal of any $k-1$ nodes, which proves this theorem.

Below we make some observations about Theorem 2 and Theorem 3. First, a major difference is that Theorem 2 can guarantee only 1 connectivity, while Theorem 3 can guarantee $k$ connectivity. This is because, in a network where each sensor is $k$-MPC, the removal of any sensor may disconnect the network. For example, in the network in Fig. 3(a), each sensor is 2-MPC. By Theorem 2, the network is 2-covered and 1-connected. However, if we remove sensor $a$, the network will be partitioned into two components. Interestingly, although the network remains 2-covered, it is no longer connected.

Second, the reverse direction of Theorem 3 may not be true. That is, if a network is $k$-covered and $k$-connected, sensors in this network may not be $k$-DPC. Fig. 3(b) shows an example in which the network is 2-covered and 2-connected. However, each node has a neighbor (with overlapping sensing range) to which there is no direct communication link.

Third, Theorem 3 is stronger than the results in [Wang et al. 2003; Zhang and Hou 2004a]. It is clear that when two sensors have overlapping sensing range, there is a direct communication link between these two sensors if the communication distance is at least twice the sensing distance. So what can be determined by [Wang et al. 2003; Zhang and Hou 2004a] can also be determined by Theorem 3. Furthermore, when the above assumption does not exist, Theorem 3 may still work while [Wang et al. 2003; Zhang and Hou 2004a] do not. For example, Theorem 3 can determine that the network in Fig. 4 is 1 covered and 1-connected, when some sensors' communication ranges are less than twice their sensing ranges. 


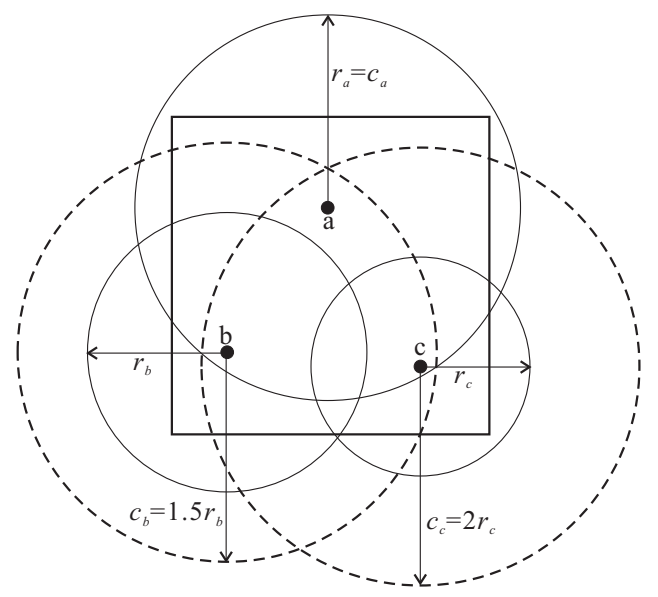

Fig. 4. An example to compare Theorem 3 with results in literature. Solid circles and dotted circles are sensors' sensing ranges and communications ranges, respectively.

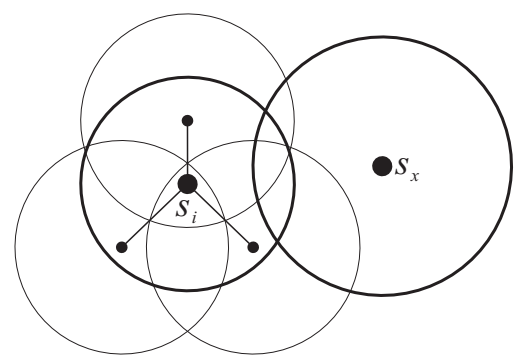

Fig. 5. An example that $k$-LDPC is looser than $k$-DPC.

\subsection{Looser Connectivity Conditions}

Definition 7. The direct neighboring set of $s_{i}$, denoted as $D N(i)$, is the set of sensors each of which has a communication link to $s_{i}$ and whose sensing region intersects with $s_{i}$ 's sensing region. Similarly, the multi-hop neighboring set of $s_{i}$, denoted as $M N(i)$, is the set of sensors each of which has a (single- or multi-hop) path to $s_{i}$ and whose sensing region intersects with $s_{i}$ 's.

Definition 8 . Consider any sensor $s_{i}$. We say that $s_{i}$ is $k$-loose-direct-neighbor-perimeter-covered, or $k$-LDPC, if $s_{i}$ is $k$-perimeter-covered by and only by nodes in $D N(i)$. Similarly, we say that $s_{i}$ is $k$-loose-multihop-neighbor-perimeter-covered, or $k$-LMPC, if $s_{i}$ is $k$-perimeter-covered by and only by nodes in $M N(i)$.

We comment that for any sensor $s_{i}, D N(i) \subseteq M N(i) \subseteq N(i)$. So the definition that $s_{i}$ is $k$-LDPC is looser than that $s_{i}$ is $k$-DPC in the sense that $k$-DPC guarantees that there is a link from $s_{i}$ to each of $N(i)$, but $k$-LDPC only guarantees that there is a link from $s_{i}$ to each of $D N(i)$. For example, consider sensor $s_{i}$ in Fig. 5. There is another sensor $s_{x}$ whose sensing range intersects with sensor $s_{i}$ 's sensing range but who has no link to $s_{i}$, i.e., $s_{x} \in N(i)-D N(i)$. Without taking $s_{x}$ into account, $s_{i}$ is 1-perimeter-covered by and only by nodes in $D N(i)$ and is thus 1-LDPC. However, $s_{i}$ is not 1-DPC since it does not 


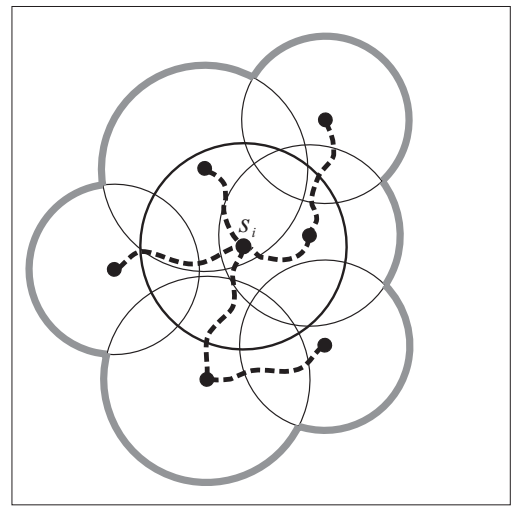

Fig. 6. Proof of the Lemma 2.

have a link to each node in $N(i)$. The definition of $k$-LMPC is looser than that of $k$-MPC in a similar sense.

LEMMA 2. If each sensor in $S$ is 1-LMPC, then the network can be decomposed into a number of connected components each of which 1-covers the sensing field $A$.

PROOF. This proof is by construction. For any sensor $s_{i}$, we try to construct a connected component which fully covers $A$. (However, the proof does not guarantee that $s_{i}$ has a path to every sensor.) If $s_{i}$ 's sensing region can fully cover $A$, the construction is completed. Otherwise, by Definition 8, nodes in $M N(i)$ must perimeter-cover $s_{i}$ 's perimeter and each has a path to $s_{i}$, as illustrated in Fig. 6. In addition, nodes in $M N(i)$ together with $s_{i}$ form a larger coverage region which is bounded by perimeters of nodes in $M N(i)$. If $A$ is already fully covered by this region, the construction is completed. Otherwise, since each sensor is 1-LMPC, we can repeat similar arguments by extending the coverage region, until the whole field $A$ is covered.

THEOREM 4. A sensor network can be decomposed into a number of connected components each of which $k$-covers $A$ iff each sensor is $k$-LMPC.

THEOREM 5. A sensor network can be decomposed into a number of $k$-connected components each of which $k$-covers $A$ if each sensor is $k$-LDPC.

The proof of Theorem 4 (respectively, Theorem 5) is similar to Theorem 2 (respectively, Theorem 3) by replacing Lemma 1 with Lemma 2. We comment that although the results of Theorem 4 and Theorem 5 do not seem to be very desirable if one only knows that there are multiple 1- or $k$-connected components in the network, this is what we have to face in practice when deploying a sensor network. An example of Theorem 4 is shown in Fig. 7. Due to relatively small communication ranges compared to sensing ranges, the network is partitioned into two connected components. However, each component still provides sufficient 1-coverage.

To summarize, Theorem 4 and Theorem 5 only guarantee that the network can be sufficiently covered by each connected component, while Theorem 2 and Theorem 3 can guarantee both coverage and connectivity of the whole network. When $D N(i)=N(i)$ or $M N(i)=N(i)$ for each sensor $s_{i}$, these theorems converge. Also observe that Theorem 4 


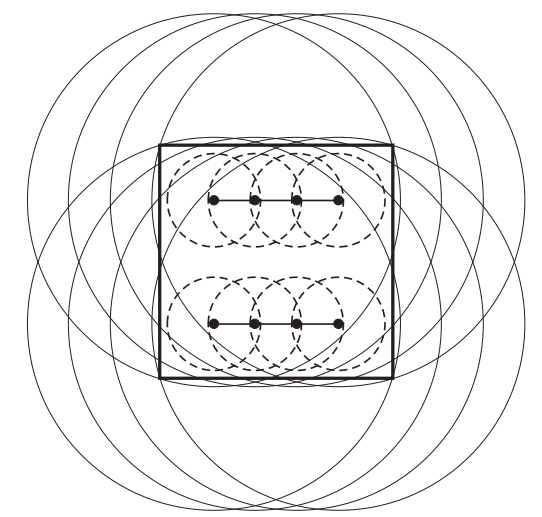

Fig. 7. An example of two connected components each of which 1-covers $A$.

and Theorem 5 are more practical because each sensor only needs to collect its reachable neighbors' information to make its decision. Most applications can be satisfied if a subset of sensors is connected and can provide sufficient coverage. The redundance caused by multiple components may be eliminated by a higher level coordinator, such as the base station, to properly schedule each component's working time such that no two components of the network are active at the same time.

\section{DISTRIBUTED COVERAGE AND CONNECTIVITY PROTOCOLS}

The quality of a sensor network can be reflected by the levels of coverage and connectivity that it offers. The above results provide us a foundation to determine, or even select, the quality of a sensor network by looking at how each sensor's perimeter is covered by its neighbors. Section 4.1 shows how to translate the above results to fully distributed coverage-and-connectivity-determination protocols. When sensors are overly deployed, the coverage and connectivity of the network may exceed our expectation. In this case, Section 4.2 proposes a distributed quality selection protocol to automatically adjust its coverage and connectivity by putting sensors into sleep mode and tuning sensors' transmission power. In Section 4.3, we show how to integrate the above results into one energy-saving protocol to prolong the network lifetime.

\subsection{Coverage and Connectivity Determination Protocols}

The goal of the protocol is to determine the levels of coverage and connectivity of the network. For a sensor to determine how its perimeter is covered, first it has to collect how its one-hop neighboring sensors' sensing regions intersect with its sensing region and calculate the level of its perimeter coverage. Periodical BEACON messages can be sent to carry sensors' location and sensing range information. On receiving such $B E A C O N$ messages, a sensor can determine who its direct neighbors are and how its perimeter is covered by them. As reviewed in Section 2, determining a sensor's perimeter coverage can be done efficiently in polynomial time [Huang and Tseng 2003]. If the level of perimeter coverage is determined to be $k$ in this step, we can say that this sensor is $k$-LDPC.

If the above level of coverage, $k$, is below our expectation, the sensor can flood a $Q U E R Y$ message to its neighbors to find out who else having overlapping sensing regions with itself. The flooding can be a localized flooding (with a certain hop limit) to save cost. Each 
sensor who receives the QUERY message has to check if its sensing region intersects with the source node's sensing region. If so, a REPLY message is sent to the source node. By so doing, the source node can calculate its level of perimeter coverage based on the received REPLY messages. If the level of perimeter coverage is determined to be $k^{\prime}$ in this step $\left(k^{\prime}\right.$ $\geq k$ ), we can say that this sensor is $k^{\prime}$-LMPC. If this value is still below our expectation, we can take an incremental approach by flooding another QUERY with a larger hop limit, until the desired level of coverage is reached or the whole network is flooded.

After the above steps, each sensor can report its exploring result to the base station or a certain centralized sensor. Then the base station can determine the coverage and connectivity levels of the network. There are three possible cases. If each sensor is at least $k$-LDPC, the network is $k$-covered and $k$-connected. If some sensors are at least $k$ LMPC while others are at least $k$-LDPC, the network is $k$-covered and 1 -connected. If there exists some sensors that are neither $k$-LDPC nor $k$-LMPC, then the network must be disconnected. In this case, it is possible that the network is still sufficiently covered but is partitioned. For example, if we remove sensor $a$ in Fig. 3(a), the network is disconnected into two parts. Although these two parts together provide 2-level coverage, since sensors are unable to exchange information, such a situation can not be determined by the network.

\subsection{Coverage and Connectivity Selection Protocols}

When sensors are overly deployed, one may want to put some sensors into sleep mode to reduce the level of coverage. One may further reduce the transmission power of sensors to reduce the network connectivity. As far as we know, the combination of these mechanisms has not been studied in the literature. In this subsection, we explore these two possibilities based on the foundations developed in Section 3.

The basic idea is as follows. Suppose that we are given a sensor network that is $k_{\text {init }}{ }^{-}$ covered and $k_{\text {init }}$-connected (this can be decided by Theorem 4 and the protocol in Section 4.1 ). If such levels of coverage and connectivity are beyond our expectation, we propose a protocol to modify the network to $k_{s}$-covered and $k_{c}$-connected such that $k_{\text {init }}$ $\geq k_{s} \geq k_{c} \geq 1$. First, in Section 4.2.1, we present a sleep protocol to reduce the network to $k_{s}$-LDPC (which means $k_{s}$-covered and $k_{s}$-connected) by putting some sensors into sleep mode. Then, in Section 4.2.2, a power control protocol is presented to reduce the network to $k_{c}$-LDPC. This results in a $k_{s}$-covered, $k_{c}$-connected network because reducing the transmission power of a sensor will not affect its sensing range.

4.2.1 The Sleep Protocol. In this protocol, each sensor only needs to know the locations and sensing regions of its two-hop neighbors that are in the active state. Two-hop neighbor information can be obtained by attaching the direct neighbor information of each sensor in its periodical BEACON messages. The information should include a sensor's location, sensing range, and current power setting. Since wireless sensor networks are typically considered static, the cost to exchange such information should be low. Suppose that the network is $k_{\text {init }}$-LDPC. The purpose of this protocol is to put some sensors into the sleep mode such that the network is at least $k_{s}$-LDPC, where $k_{\text {init }} \geq k_{s}$. For each sensor $S_{x}$ that intends to go to sleep, it will execute the following procedure:

(1) For each $s_{y}$ that is a direct neighbor of $s_{x}$ such that $s_{x}$ and $s_{y}$ have overlapping in their sensing regions, let $p\left(s_{x}, s_{y}\right)$ be the perimeter of $s_{y}$ 's sensing range that is covered by $s_{x}$ 's sensing range. Sensor $s_{x}$ then calculates the level of coverage of $p\left(s_{x}, s_{y}\right)$. If the level of coverage is at least $k_{s}+1$, then $s_{x}$ is a candidate. 


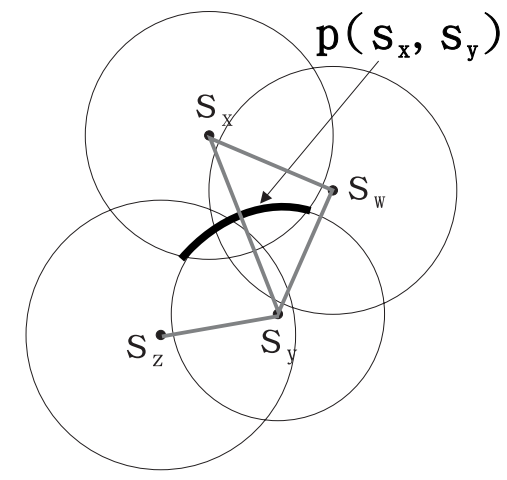

Fig. 8. An example of the Sleep Protocol. Sensor $s_{x}$ is a candidate with respect to sensor $s_{y}$.

(2) If $s_{x}$ is a candidate for each $s_{y}$ that is a direct neighbor of $s_{x}$, then $s_{x}$ is eligible to go to the sleep mode. Then $s_{x}$ waits for a random backoff time $T_{\text {rand }}$ and overhears if there is any other sleeping request. (One possibility is to set $T_{\text {rand }}$ according to $s_{x}$ 's remaining energy.) If any sleeping request is heard, $s_{x}$ will go back to step 1 , hold for another random period, and try again. Otherwise, $s_{x}$ will send a SLEEP message to each of its neighbors and wait for their responses by setting up a timer $T_{s}$.

(3) Each $s_{y}$ which is a neighbor of $s_{x}$ can reply a GRANT-SLEEP message to $s_{x}$ if it has no pending grant currently. Otherwise, a REJECT-SLEEP message is replied. Note that to avoid erroneously putting too many sensors into sleep and to maintain synchronization, a sensor can have at most one pending grant at one time. Specifically, a GRANTSLEEP message is clear from the pending status once a CONFIRM/WITHDRAW message is received (see step 4 below).

(4) If $s_{x}$ can collect a GRANT-SLEEP message from each of its neighbors, $s_{x}$ broadcasts a CONFIRM message to its neighbors and then goes to sleep. If any REJECT-SLEEP message is received or the timer $T_{s}$ expires, $s_{x}$ broadcasts a WITHDRAW message to its neighbors.

Note that in the above step $1, s_{x}$ needs to know all direct neighbors of sensor $s_{y}$. Since $s_{x}$ and $s_{y}$ are direct neighbors, these sensors are $s_{x}$ 's two-hop neighbors. Fig. 8 shows an example of the above protocol. If $s_{x}$ intends to go to sleep, it will check the perimeter $p\left(s_{x}, s_{y}\right)$ (shown in thick line). Since $p\left(s_{x}, s_{y}\right)$ is also covered by $s_{z}$ and $s_{w}$. If the target coverage is $k_{s}=1$, then $s_{x}$ is a candidate with respect to $s_{y}$. Also note that the timer $T$ is necessary because we assume an unreliable broadcast.

4.2.2 The Power Control Protocol. The power control protocol is aim to reduce the transmission power of sensors to save energy. Since this operation does not affect the sensing unit(s), the sensing capability of sensors (and thus the level of coverage of the network) is not reduced. Suppose that the network is $k_{s}$-LDPC. The purpose of this protocol is to reduce some sensors' transmission power to make the network at least $k_{c}$-LDPC, where $k_{s}$ $\geq k_{c}$. This results in a $k_{s}$-covered, $k_{c}$-connected network.

This protocol assumes that each sensor knows the information of its two-hop neighbors. For sensor $s_{x}$ which intends to reduce its transmission powers, it executes the following procedure:

ACM Journal Name, Vol. V, No. N, M 20YY. 


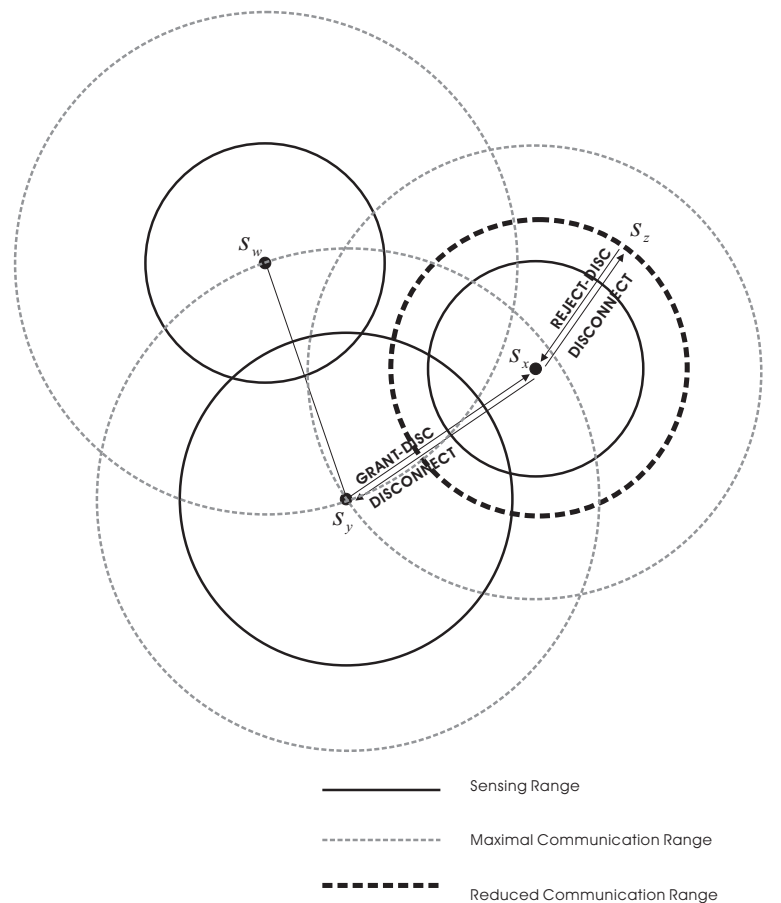

Fig. 9. A power control protocol example.

(1) Let $s_{y}$ be the direct neighbor of $s_{x}$ that is farthest from $s_{x}$. Sensor $s_{x}$ then computes the perimeter coverage of the segments $p\left(s_{x}, s_{y}\right)$ and $p\left(s_{y}, s_{x}\right)$. If both segments are at least $\left(k_{c}+1\right)$-LDPC, $s_{x}$ is allowed to conduct power control. Then $s_{x}$ waits for a random backoff time $T_{\text {rand }}$ and overhears if there is any other disconnecting request. If any request is heard, $s_{x}$ will go back to step 1, hold for another random period, and try again. Otherwise, $s_{x}$ will send a DISCONNECT message to $s_{y}$ and wait for its response by setting up a timer $T_{p}$.

(2) On receipt of $s_{x}$ 's disconnecting request, if $s_{y}$ has no pending disconnecting request currently, $s_{y}$ can reply a GRANT-DISC message to $s_{x}$. Otherwise, a REJECT-DISC is replied. Note that a DISCONNECT message is clear from the pending status once a GRANT-DISC / REJECT-DISC message is received or the timer $T_{p}$ expires.

(3) If a GRANT-DISC message is received, $s_{x}$ can reduce its transmission power such that only its second farthest direct neighbor is covered and go back to step 1 to try to further reduce its transmission power. Otherwise, a REJECT-DISC message will stop $s_{x}$ from reducing its transmission power.

Note that in the above protocol, sensor $s_{y}$ may not be able to reduce its transmission power even if $s_{x}$ successfully reduces its power. This is because $s_{y}$ may need to maintain connectivity with other neighbors that are farther away than $s_{x}$. Another comment is that here we choose to let $s_{x}$ reduce its power step by step. The concern here is for fairness.

Fig. 9 shows an example. Initially, the network is 2-covered and 2-connected (i.e., $k_{i n i t}=2$ ). We only consider sensor $s_{x}$ and its two neighbors $s_{y}$ and $s_{z}$. We will dis- 
connect the communication link between $s_{x}$ and its farthest direct neighbor, $s_{y}$, by power control. First, $s_{x}$ examines its intersection with $s_{y}$. Both segments $p\left(s_{x}, s_{y}\right)$ and $p\left(s_{y}, s_{x}\right)$ are 2-LDPC, so $s_{x}$ sends a DISCONNECT message to $s_{y}$, which will agree by replying a GRANT-DISC message. Then $s_{x}$ can reduce its transmission power to the level that can reach the next farthest neighbor $s_{z}$. Next, $s_{x}$ examines its intersection with $s_{z}$. Both segments $p\left(s_{x}, s_{z}\right)$ and $p\left(s_{z}, s_{x}\right)$ are 2-LDPC, so $s_{x}$ sends a DISCONNECT message to $s_{z}$. Suppose that $s_{z}$ has a pending disconnecting request currently, it will reply a REJECTDISC message to $s_{x}$. Then $s_{x}$ stops its procedure. Note that in the above scenario, $s_{y}$ may not necessarily reduce its transmission power even if it grants $s_{x}$ 's request to reduce power. For example, $s_{y}$ may not be able to reduce its power because $s_{w}$ wants to remain connected with $s_{y}$. In order to maintain connectivity with $s_{w}, s_{y}$ can still reach $s_{x}$. This results in an asymmetric link between $s_{x}$ and $s_{y}$ (i.e., the transmission power of $s_{x}$ cannot reach $s_{y}$, but the transmission power of $s_{y}$ can reach $s_{x}$ ). Therefore, only $s_{x}$ can benefit from the transmission power.

\subsection{An Integrated Energy-Saving Protocol}

In Fig. 10, we show how to integrate the above coverage and connectivity determination protocol, sleep protocol, and power control protocol together into one protocol. The purpose is to save energy while maintaining the quality of the network. Basically, these subprotocols are executed in that order. We assume that the goal is to achieve a $k_{s^{-}}$-covered, $k_{c^{-}}$ connected network, where $k_{s} \geq k_{c}$. In particular, we set up two timers, one called $T_{\text {sleep }}$ for sleeping sensors to wake up themselves, and one called $T_{\text {cycle }}$ for sensors to re-check their local coverage and connectivity (this is to prevent neighboring sensors from running out of batteries, thus resulting in a network weaker than $k_{s}$-covered and $k_{c}$-connected). Also, a new HELP message is designed for sensors to call others' assistance to increase the coverage and connectivity of the network (if possible) when some sensors run out of energy. Note that whenever a sensor goes to the initial state, it will use the largest transmission power to determine its local network coverage and connectivity. For example, this applies to a sensor when it receives a HELP message under a reduced transmission power status.

\section{SIMULATION RESULTS}

In this section, we present two sets of simulation experiments. Experiment 1 tests the network coverage and connectivity at different sensing ranges and communication ranges. Experiment 2 evaluates the performance of the proposed energy-saving protocol.

\subsection{Experiment 1: Coverage and Connectivity}

We have developed a simulator to compare the network coverage and connectivity calculated by Theorem 5 and by an exhausted search algorithm. All results in this section are from averages of at least 100 runs. The simulation environment is a 100x100 square area, on which sensors are randomly deployed. The sensing range and communication range of each sensor are uniformly distributed in certain ranges.

Fig. 11 shows the coverage and connectivity under different communication ranges. Note that Theorem 5 may not be able to find the exact coverage and connectivity levels because it only relies on local information. Our goal is to compare the results obtained by Theorem 5 (which implies coverage as well as connectivity) against the minimum of the actual coverage and actual connectivity obtained by an exhausted search. So Fig. 11(a) 


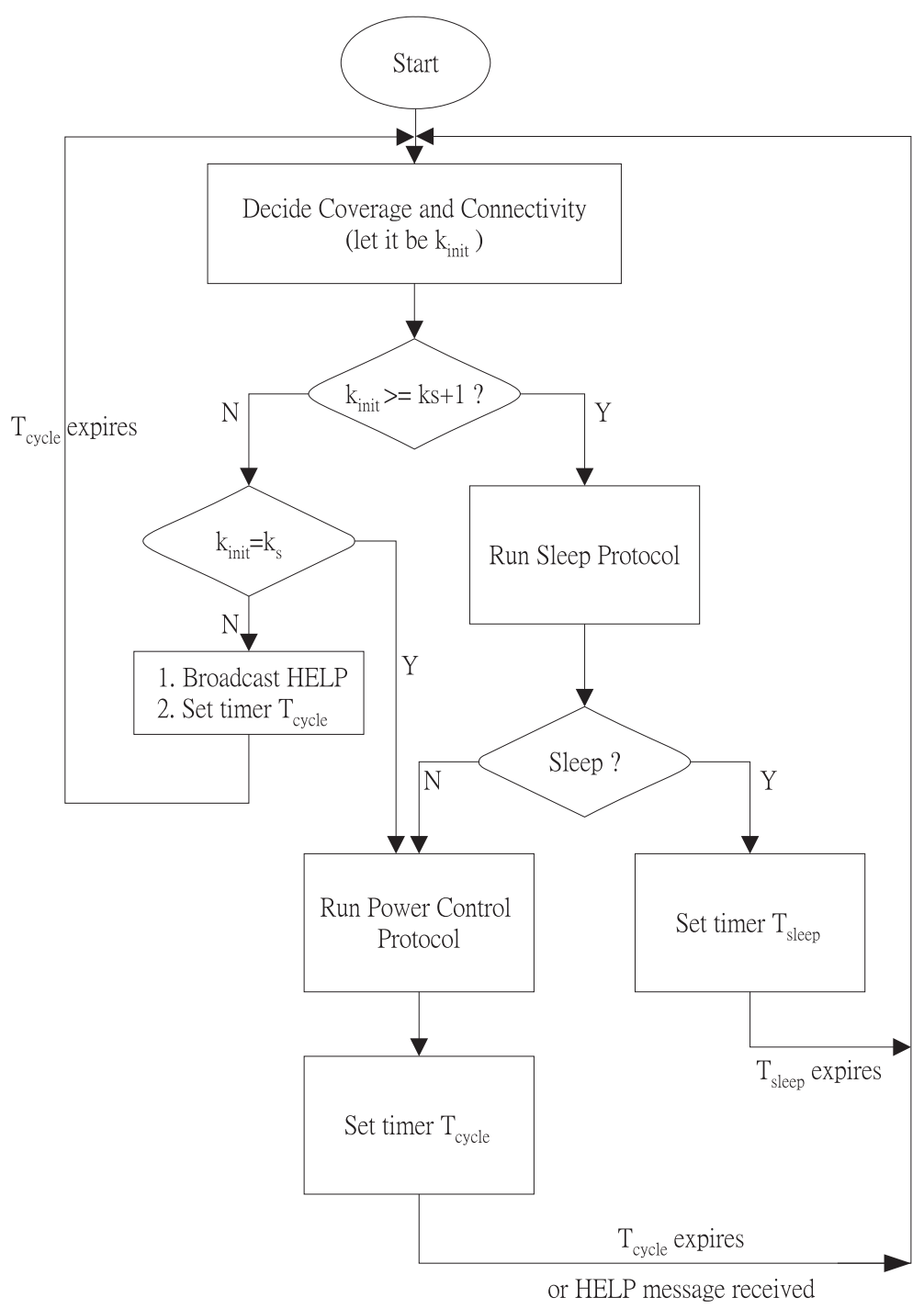

Fig. 10. An integrated energy-saving protocol.

represents an ideal situation because what are found by Theorem 5 match closely with the actual values. The gaps increase as we move to Fig. 11(b), (c), and (d). This is because the ratios of average communication range to average sensing range are reduced, which means that a sensor may not be able to know the existence of another sensors which have overlapping with its own sensing range if it only examines its direct neighbors. So a certain degrees of coverage and connectivity are not discovered by Theorem 5 .

Next, we keep the sensing ranges fixed, but change the communication ranges variations. Fig. 12 shows the coverage and connectivity in a 300-nodes network when we vary the mean and variation of communication ranges. Note that in each point of Fig. 12(a), 


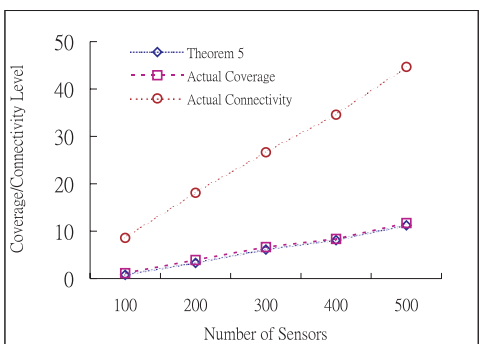

(a) Sensing range $=15 \sim 25$, Communication range $=30 \sim 50$

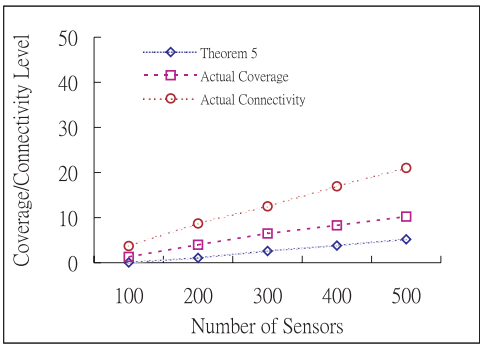

(c) Sensing range $=15 \sim 25$, Communication range $=20 \sim 40$

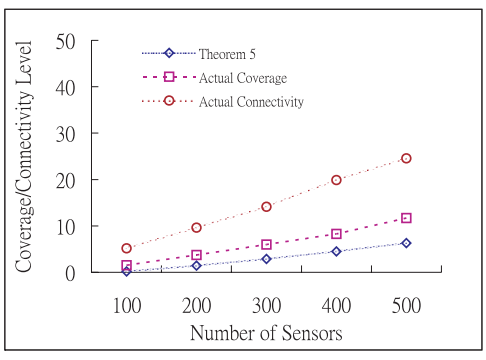

(b) Sensing range $=15 \sim 25$, Communication range $=20 \sim 60$

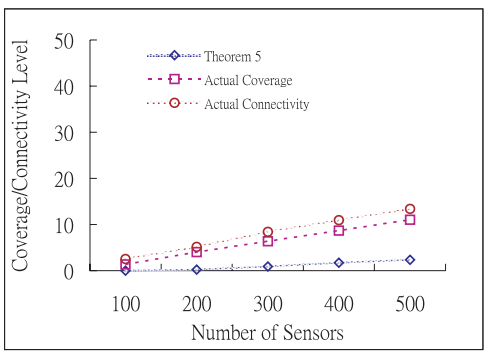

(d) Sensing range $=15 \sim 25$, Communication range $=15 \sim 45$

Fig. 11. Network coverage and connectivity under different communication ranges.

sensors' communication ranges have no variation, while in each point of Fig. 12(b), the variation range is 20. As can be seen, although in both cases Theorem 5 finds about the same values of coverage and connectivity, since the actual connectivity reduces, Theorem 5 actually matches closer to the actual situations in the case of Fig. 12(b). In Fig. 13, we conduct the similar simulation by keeping the communication ranges unchanged but changing the mean and variation of sensing ranges. The trend is similar - Theorem 5 matches closer to the actual situations when there are larger variations in sensing ranges. Also, by comparing Fig. 12 and Fig. 13, we observe that the gaps reduce when the ratios of average communication range to average sensing range increase. The reason is that as the ratio increases, a sensor is able to collect more information about its neighborhood.

\subsection{Experiment 2: Network Life Time}

This section verifies our integrated energy-saving protocol for prolonging network lifetime while ensuring both coverage and communication quality. We consider three performance metrics: number of alive nodes, coverage level, and connectivity level. In these experiments, there are 300 sensors randomly deployed in a 100x100 square area with sensing range $=15 \sim 25$ units, communication range $=30 \sim 50$ units, and initial energy $=8000$ $\sim 12000$ units (all in a uniform distribution). Our goal is to achieve a $k_{s}$-covered and $k_{c^{-}}$ connected network. We sample the network status every 10 seconds. We assume a constant traffic rate for each sensor, and the energy cost of each transmission is proportional to a sensor's transmission range. The energy cost for sensing is also proportional to a sensor's sensing range [Lu et al. 2005]. Therefore, for each sensor $s_{i}$, the energy consumed every second is proportional to the sum of its sensing range $r_{i}$ and its current communication 


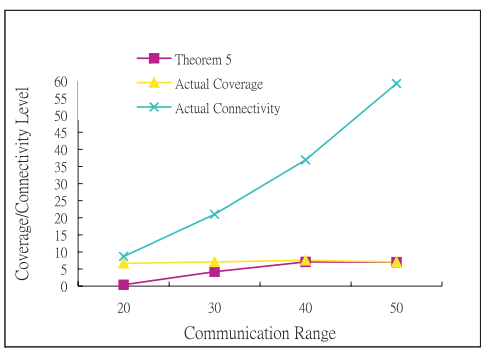

(a) Sensing Range $=10 \sim 30$

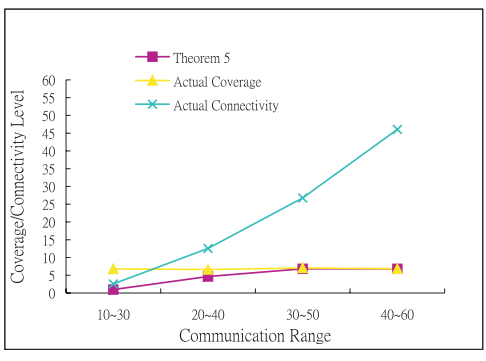

(b) Sensing Range $=10 \sim 30$

Fig. 12. Network coverage and connectivity under different means and variations of communication ranges.

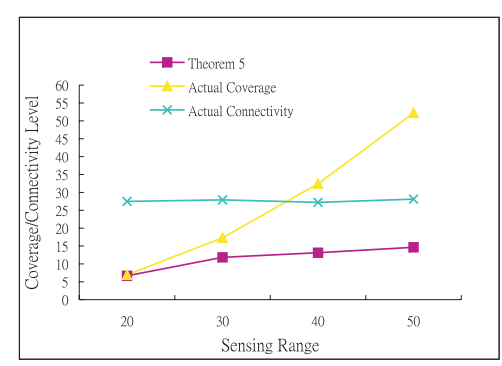

(a) Communication Range $=30 \sim 50$

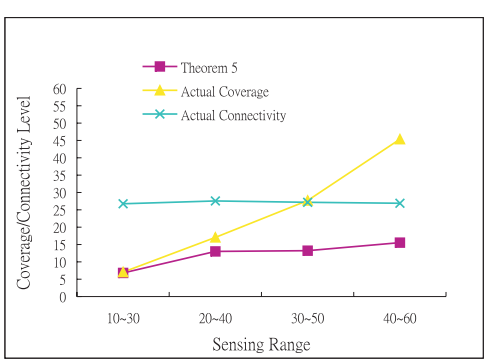

(b) Communication Range $=30 \sim 50$

Fig. 13. Network coverage and connectivity under different means and variations of sensing ranges.

range $c_{i}$. Although this is a simplified assumption, if the energy cost of each transmission is proportional to a sensor's transmission distance raised to a factor of 2 or 4, our power control scheme should demonstrate even more saving in energy consumption.

Two versions of protocols are evaluated, one with the Sleep protocol only and the other with Sleep+Power Control protocol (denoted by Sleep+PC). We compare our results against a naive protocol, where all sensors are always active, and the CCP+SPAN protocol [Wang et al. 2003]. CCP (Coverage Configuration Protocol) is a protocol that can dynamically configure a network to achieve guaranteed degrees of coverage and connectivity if sensors' communication ranges are no less than twice their sensing ranges. If sensors' communication ranges are less than twice their sensing ranges, [Wang et al. 2003] suggests to integrate CCP with SPAN, which is a decentralized protocol that tries to conserve energy by turning off unnecessary nodes while maintaining a communication backbone composed of active nodes.

Fig. 14(a) shows the number of alive sensors when the goal is to maintain a 2-covered and 1-connected network. In the naive protocol, because nodes are always active, the number of alive sensors drops sharply at around $150 \mathrm{sec}$. Sensors in CCP+SPAN protocol fail at a slower speed. Both Sleep and Sleep+PC protocols can significantly reduce the 


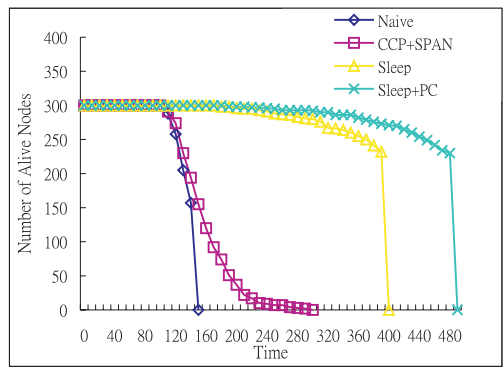

(a) $\mathrm{k}_{\mathrm{s}}=2, \mathrm{k}_{\mathrm{c}}=1$

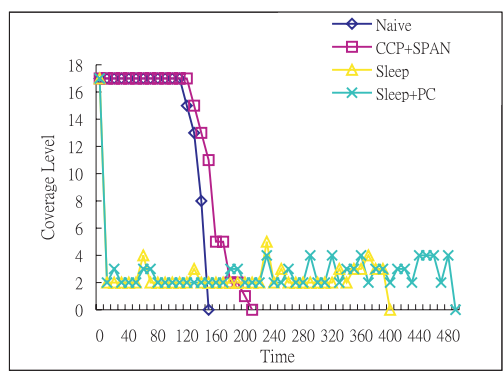

(b) $\mathrm{ks}=2, \mathrm{kc}=$

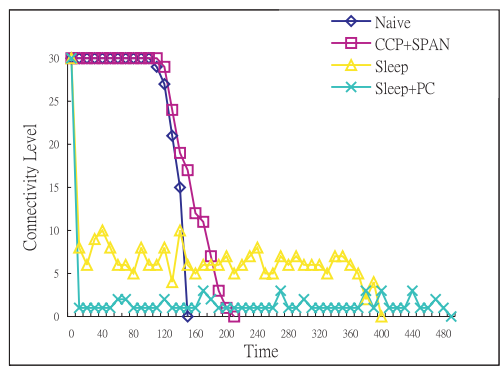

(c) $\mathrm{ks}=2, \mathrm{kc}=1$

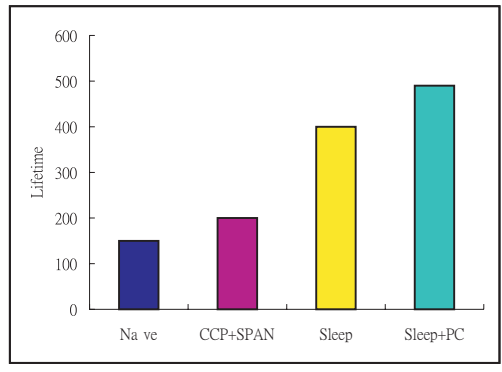

(d) $\mathrm{ks}=2, \mathrm{kc}=1$

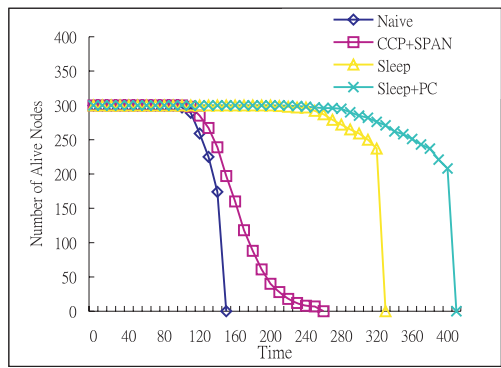

(e) $\mathrm{ks}=3, \mathrm{kc}=2$

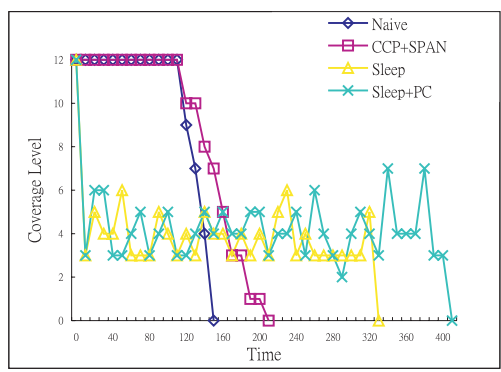

(f) $\mathrm{ks}=3, \mathrm{kc}=2$

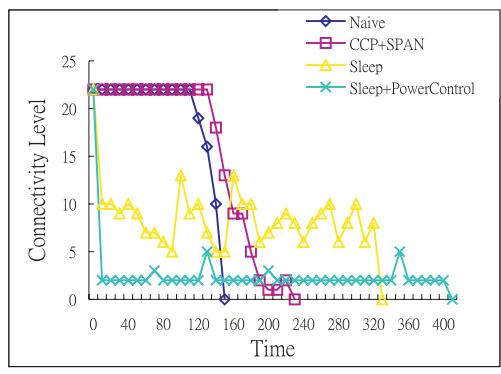

(g) $\mathrm{ks}=3, \mathrm{kc}=2$

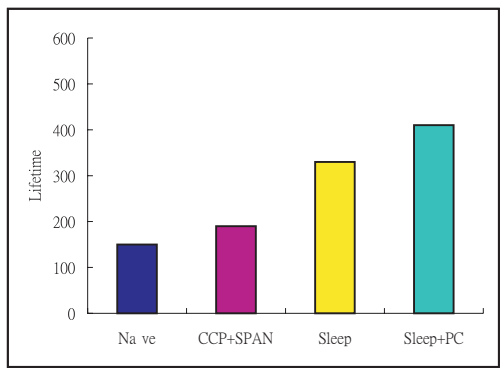

(h) $\mathrm{ks}=3, \mathrm{kc}=2$

Fig. 14. Comparisons of the naive, $\mathrm{CCP}+\mathrm{SPAN}$, Sleep, and Sleep+PC protocols.

ACM Journal Name, Vol. V, No. N, M 20YY. 


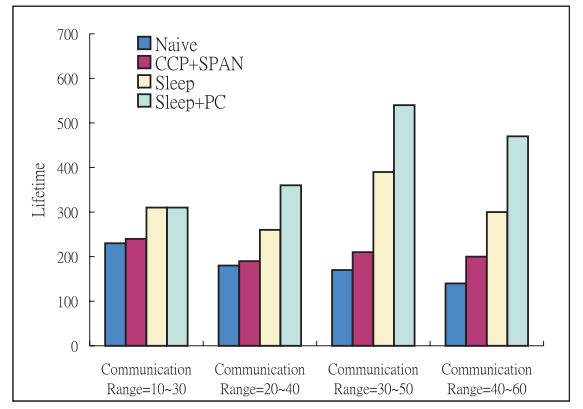

(a) $\mathrm{ks}=2, \mathrm{kc}=1$

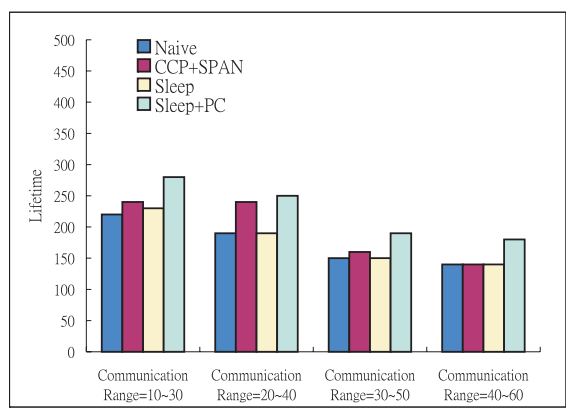

(b) $\mathrm{ks}=3, \mathrm{kc}=2$

Fig. 15. Network lifetime under different communication ranges (Sensing Range $=15 \sim$ 25).
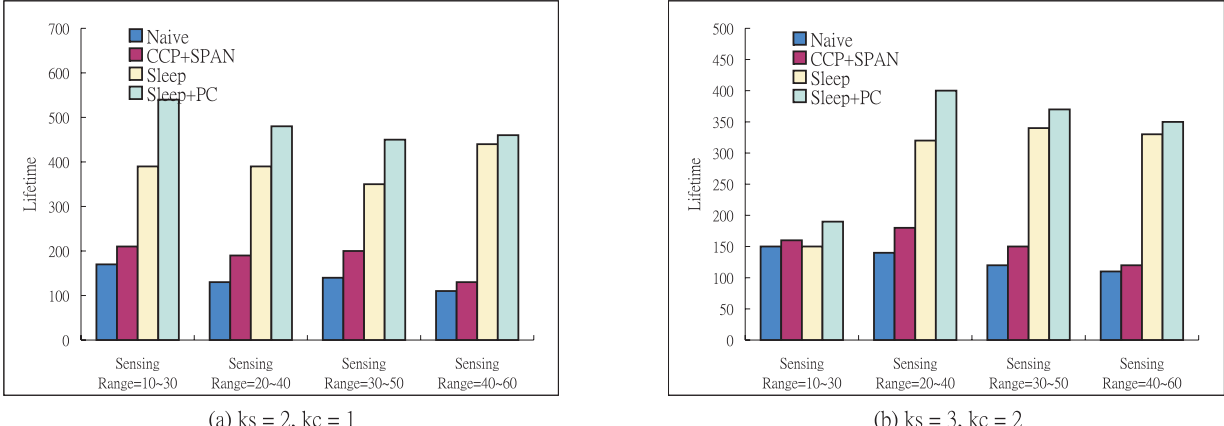

Fig. 16. Network lifetime under different sensing ranges (Communication Range $=30 \sim$ $50)$.

rate that sensors fail. Overall, Sleep+PC performs the best. This can be explained by the levels of coverage and connectivity provided by a protocol, as shown in Fig. 14(b) and Fig. 14(c). There is too much redundancy in coverage and connectivity in both the naive and CCP+SPAN protocols. The Sleep protocol maintains the level of coverage pretty well, but the level of connectivity is still much higher than our expectation. Only Sleep+PC can maintain the best-fit coverage and connectivity levels. This justifies the usefulness of adopting power control to adjust the communication topology of the network. Fig. 14(d) shows the network lifetime, which is defined as the time before the levels of coverage and connectivity drop below our expectations. The lifetime of the naive protocol is around 150 sec. The lifetime of CCP+SPAN is around $200 \mathrm{sec}$. The Sleep and Sleep+PC protocols can significantly prolong network lifetime to around 340 and 410 sec., respectively. Fig. 14(e), $(\mathrm{f}),(\mathrm{g})$, and $(\mathrm{h})$ are from similar experiments when the goal is to maintain a 3-covered and 2-connected network. The trend is similar.

In the following, only the network lifetime is shown. Fig. 15 shows the network lifetime under the same sensing range $(15 \sim 25)$ but different communication ranges. In all 


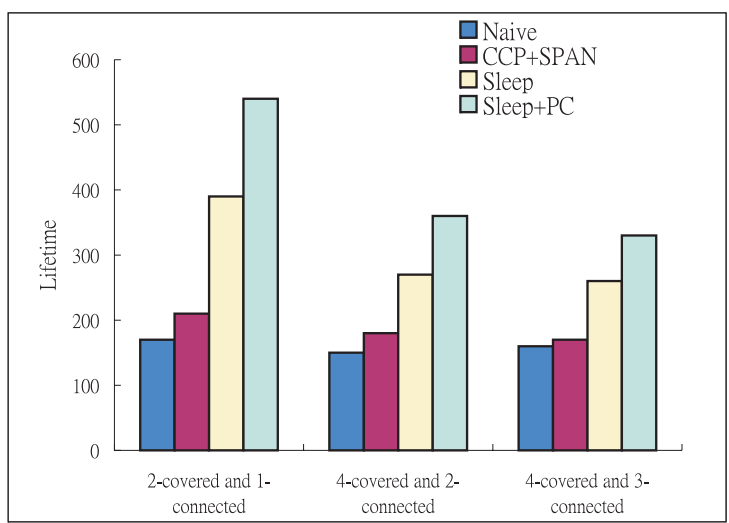

Fig. 17. Network lifetime under different coverage and connectivity requirements (Sensing Range $=15 \sim 25$ and Communication Range $=30 \sim 50$ ).

situations, Sleep+PC performs the best. In fact, when the communication range increases, the gaps between Sleep+PC and other protocols enlarge relatively. So our power control scheme can effectively reduce network connectivity and prolong network lifetime. Basically, our schemes can perform better when sensors have larger communication ranges. This is because sensors with larger transmission ranges can find more neighbors, collect more necessary information needed for making sleeping and power-controlling decisions, and thus have higher chance to go to sleep and/or shrink their powers. Besides, it is obvious that power control can more effectively reduce network connectivity if sensors' initial communication ranges are larger. However, how much Sleep+PC can outperform other schemes also relies on the network density and the level of coverage and connectivity to be achieved. If $k_{s}$ and $k_{c}$ are closer to $k_{\text {init }}$, our scheme is less effective. Fig. 16 shows the similar experiments under the same communication range $(30 \sim 50)$ but different sensing ranges. In Fig. 17, we further test under different coverage and connectivity requirements. Around 1 to 2 times more lifetime can be seen when comparing Sleep+PC to CCP+SPAN.

\section{CONCLUSIONS AND FUTURE WORK}

We have proposed fundamental theorems for determining the levels of coverage and connectivity of a sensor network. Earlier works are all based on stronger assumptions that the sensing distances and communication distances of sensors must satisfy some relations. We study this issue under an arbitrary relationship between sensing and communication ranges. Based on the proposed theorems, we have developed distributed protocols for determining the levels of coverage and connectivity of a sensor network and even for adjusting a sensor network to achieve the expected levels of coverage and connectivity. The approaches that we take are to put some sensors into the sleep mode and to reduce some sensors' transmission power. As far as we know, the combination of these mechanisms has not been well studied in this field, especially when coverage and connectivity issues are concerned. In our work, a deterministic model is used to formulate sensors' sensing and communication ranges. In reality, these values may follow a probabilistic model (such as 
a sensor can successfully detect an object in a distance $d$ with a probability $\operatorname{prob}(d)$ ). The coverage-connectivity-combined issue still requires further investigation in this direction.

\section{REFERENCES}

Abrams, Z., Goel, A., AND Plotkin, S. 2004. Set K-cover algorithms for energy efficient monitoring in wireless sensor networks. In Int'l Symp. on Information Processing in Sensor Networks (IPSN). 424-432.

Bahl, P. And PAdmanabhan, V. N. 2000. RADAR: An in-building RF-based user location and tracking system. In IEEE INFOCOM. 775-784.

BRAGINSKY, D. AND ESTRIN, D. 2002. Rumor routing algorithm for sensor networks. In ACM Int'l Workshop on Wireless Sensor Networks and Applications (WSNA).

Burkhart, M., von Rickenbach, P., Wattenhofer, R., And Zollinger, A. 2004. Does topology control reduce interference? In ACM Int'l Symp. on Mobile Ad Hoc Networking and Computing (MobiHOC). $9-19$.

CHEN, B., JAMIESON, K., BALAKRISHNAN, H., AND MORRIS, R. 2002. Span: an energy-efficient coordination algorithm for topology maintenance in ad hoc wireless networks. ACM Wireless Networks 8, 5 (Sep.), 481494.

Ganesan, D., Govindan, R., Shenker, S., And Estrin, D. 2001. Highly resilient, energy efficient multipath routing in wireless sensor networks. ACM Mobile Comput. and Commun. Review 5, 4 (Oct.), 11-25.

GuPTA, H., DAS, S. R., AND GU, Q. 2003. Connected sensor cover: Self-organization of sensor networks for efficient query execution. In ACM Int'l Symp. on Mobile Ad Hoc Networking and Computing (MobiHOC). $189-200$.

Heinzelman, W. R., Chandrakasan, A., And Balakrishnan, H. 2000. Energy-efficient communication protocols for wireless microsensor networks. In Hawaii Int'l Conf. on Systems Science (HICSS).

HuAng, C.-F. AND TSEng, Y.-C. 2003. The coverage problem in a wireless sensor network. In ACM Int'l Workshop on Wireless Sensor Networks and Applications (WSNA). 115-121.

HuAng, C.-F., Tseng, Y.-C., AND Lo, L.-C. 2004. The coverage problem in three-dimensional wireless sensor networks. In IEEE Global Telecommunications Conference (Globecom).

InANC, M., MAGdon-ISMAIL, M., AND YENER, B. 2003. Power optimal connectivity and coverage in wireless sensor networks. Tech. Rep. TR-03-06, Department of Computer Science, Rensselaer Polytechnic Institute. July.

LI, N. AND HOU, J. C. 2004. FLSS: A fault-tolerant topology control algorithm for wireless networks. In ACM Int'l Conf. on Mobile Computing and Networking (MobiCom).

LI, X.-Y., WAN, P.-J., AND FRIEDER, O. 2003. Coverage in wireless ad hoc sensor networks. IEEE Trans. Comput. 52, 6 (June), 753-763.

Lu, M., Wu, J., CARDEI, M., AND Li, M. 2005. Energy-efficient connected coverage of discrete targets in wireless sensor networks. In Int'l Conf. on Computer Networks and Mobile Computing (ICCNMC).

Meguerdichian, S., Koushanfar, F., Potkonjak, M., and SRivastava, M. B. 2001. Coverage problems in wireless ad-hoc sensor networks. In IEEE INFOCOM. 1380-1387.

Meguerdichian, S., Koushanfar, F., Qu, G., And PotKonjak, M. 2001. Exposure in wireless ad-hoc sensor networks. In ACM Int'l Conf. on Mobile Computing and Networking (MobiCom). 139-150.

Pottie, G. J. And Kaiser, W. J. 2000. Wireless integrated network sensors. Commun. ACM 43, 5 (May), $51-58$.

Savvides, A., Han, C.-C., And Strivastava, M. B. 2001. Dynamic fine-grained localization in ad-hoc networks of sensors. In ACM Int'l Conf. on Mobile Computing and Networking (MobiCom). 166-179.

Shak Kottai, S., SRIKAnt, R., AND Shroff, N. 2003. Unreliable sensor grids: coverage, connectivity and diameter. In IEEE INFOCOM. 1073 - 1083.

Shin, E., Cho, S.-H., ICKes, N., Min, R., Sinha, A., WANG, A., And Chandrakasan, A. 2001. Physical layer driven protocol and algorithm design for energy-efficient wireless sensor networks. In ACM Int'l Conf. on Mobile Computing and Networking (MobiCom). 272-287.

Sohrabi, K., Gao, J., Ailawadhi, V., AND Pottie, G. J. 2000. Protocols for self-organization of a wireless sensor network. IEEE Personal Commun. 7, 5 (Oct.), 16-27.

TiAn, D. AND GEORGANAS, N. D. 2003. A node scheduling scheme for energy conservation in large wireless sensor networks. Wireless Commun. and Mobile Comput. (WCMC) 3, 271-290.

ACM Journal Name, Vol. V, No. N, M 20YY. 
Tseng, Y.-C., KuO, S.-P., LeE, H.-W., AND HuANG, C.-F. 2003. Location tracking in a wireless sensor network by mobile agents and its data fusion strategies. In Int'l Symp. on Information Processing in Sensor Networks (IPSN).

Veltri, G., HuAng, Q., QU, G., ANd PotKonjaK, M. 2003. Minimal and maximal exposure path algorithms for wireless embedded sensor networks. In ACM Int'l Conf. on Embedded Networked Sensor Systems (SenSys). $40-50$.

Wang, X., Xing, G., Zhang, Y., Lu, C., Pless, R., And Gill, C. 2003. Integrated coverage and connectivity configuration in wireless sensor networks. In ACM Int'l Conf. on Embedded Networked Sensor Systems (SenSys). 28-39.

Wattenhofer, R., LI, L., BAhL, P., AND WANG, Y.-M. 2001. Distributed topology control for power efficient operation in multihop wireless ad hoc networks. In IEEE INFOCOM. 1388 - 1397.

Woo, A. AND Culler, D. E. 2001. A transmission control scheme for media access in sensor networks. In ACM Int'l Conf. on Mobile Computing and Networking (MobiCom). 221-235.

Xu, Y., Heidemann, J., AND EstRin, D. 2001. Geography-informed energy conservation for ad hoc routing. In ACM Int'l Conf. on Mobile Computing and Networking (MobiCom). 70-84.

Yan, T., He, T., And Stankovic, J. A. 2003. Differentiated surveillance for sensor networks. In ACM Int'l Conf. on Embedded Networked Sensor Systems (SenSys). 51-62.

Ye, W., Heidemann, J., AND EstRin, D. 2002. An energy-efficient MAC protocol for wireless sensor networks. In IEEE INFOCOM. 1567-1576.

ZHANG, H. AND HOU, J. C. 2004a. Maintaining sensing coverage and connectivity in large sensor networks. In NSF International Workshop on Theoretical and Algorithmic Aspects of Sensor, Ad Hoc Wireless, and Peerto-Peer Networks.

ZHANG, H. AND HOU, J. C. 2004b. On deriving the upper bound of lifetime for large sensor networks. In ACM Int'l Symp. on Mobile Ad Hoc Networking and Computing (MobiHOC). 121 - 132.

Received MM YY; accepted MM YY 